\title{
Transmitting-Collision Tradeoff in Cognitive Radio Networks: A Flexible Transmitting Approach
}

\author{
Hong Du, Zaixue Wei, Lu Ye, Yu Wang and Dacheng Yang \\ Wireless Theories and Technologies Lab (WT\&T) \\ Beijing University of Posts and Telecommunications (BUPT) \\ Beijing, China \\ duhong929@gmail.com
}

\begin{abstract}
In cognitive radio networks, in order to avoid collision to primary user (PU), cognitive radio (CR) user ought to sense the spectrum periodically to certify that $\mathrm{PU}$ is absent. However, the collision to PU also occurs due to imperfect sensing or PU's returning when $C R$ user is transmitting. In this paper, we study a transmitting-collision tradeoff problem in cognitive radio networks. In particular, we investigate a flexible data transmitting scheme so as to minimize the collision to PU under the probability of detection constrains. In addition, by jointly considering both $C R$ user's traffic and PU's state, the probability of collision is derived in detail. Finally, numerical results indicate that the proposed approach can give a superior performance than the fixed frame duration mechanism. The probability of collision for the proposed scheme is lower than conventional mechanism obviously. The conclusion is that the larger traffic parameter of CR user leads to higher collision to PU. By increasing the probability of detection can reduce the collision to $\mathbf{P U}$.
\end{abstract}

Keywords-cognitive radio; transmitting-collision tradeoff; spectrum sensing; flexible data transmitting

\section{INTRODUCTION}

Nowadays, the spectrum resource is shortage for the increasing wireless communication services; however, evidenced by the measurements of Federal Communications Commission (FCC), more than $70 \%$ of the licensed spectrum is rarely utilized continuously across time and space [1]. Due to it is possible to access the licensed spectrum opportunistically and make use of the idle spectrum, cognitive radio (CR) technology is paid great attention for the moment [2]. Nevertheless, the data transmission of CR users will bring the collision to the primary user (PU) when it returns. Consequently, in order to avoid the collision to PUs, CR user has to sense the spectrum channel periodically to ensure the absences of the PU and transmit data later in this period.

Recently, the design of an optimal spectrum sensing strategy has received considerable attention. The common design objective of optimal sensing is to maximize the network throughput by minimizing the sensing overhead under certain performance constraints, e.g., interference to the primary users. To this end, various sensing-related performance tradeoffs have been exploited in many different contexts, such as sensing-throughput tradeoff [3-8], overhead-throughput tradeoff [9] and sensing-access tradeoff [10]. In particular, Liang et al. in [3] formulated a problem to design the sensing duration to maximize the throughput of the cognitive system under the detection probability constraint. Moreover, in order to maximize the spectrum utilization efficiency, a tradeoff between sensing time and data transmission is investigated in [11]. As in [12], the authors propose a novel cognitive radio system that overcomes the sensing-throughput tradeoff by performing spectrum sensing and data transmission at the same time, which maximizes both the sensing time and the throughput of the cognitive radio network. The proposed scheme exhibits improved throughput and sensing capabilities, but without considering the collision to PU. And in [13], a collision-utilization tradeoff problem is formulated, it considers the CR's traffic, but the data transmitting time is still fixed.

These strategies are studied to improve the network throughput significantly by exploiting various tradeoffs with regard to the design of sensing parameters. Despite its importance, however, the impact of the PU's collision has received far less attention. Intuitively, while transmitting for a longer period of time will allow CR users to obtain more throughputs in cognitive radio networks, but it will also result in more collision to PU. However, most current sensing tradeoff schemes do not consider the impact to PU's quality of service (QoS). In addition, existing spectrum sensing mechanism mainly exploits the fixed frame duration aiming at the maximum access time of $\mathrm{CR}$, but without considering the traffic of CR users.

The main contributions of this paper are described as follows. First, we study the tradeoff problem between CR user's transmitting and collision to PU. In particular, we formulate an optimization problem to minimize the collision to PU while maintaining the probability of detection above a certain lower bound. Second, we propose a flexible data transmitting approach by considering the CR user's traffic model which follows an exponential distribution. In addition, we discuss two cases of collision when CR user is transmitting. Furthermore, we derive a closed-form expression of the probability of collision for the proposed method. Last, using computer simulations, it is shown that the presented scheme significantly decreases the collision to the PU.

This work is supported by the Fundamental Research Funds for the Central Universities. 
The rest of the paper is organized as follows: In Section II, system model is provided and problem formulation is described. In Section III, we derive the probability of collision for conventional and proposed scheme, respectively. And then, numerical simulations are presented in Section IV. Finally, the conclusions are drawn in Section V.

\section{SYSTEM MODEL}

Usually, the spectrum state of PU is divided into two types: ON and OFF state, which denote whether the PU exists or not. Frame duration is denoted by $T_{p}$, which is composed of sensing time $T_{s}$ and data transmitting time $T_{d}$. In fact, CR user does not always use the spectrum; therefore, it is unreasonable to study the collision avoidance to PU while considering CR user always transmitting data. Hence, a flexible data transmitting scheme is investigated, we assumed that the spectrum usage state of CR user follows an exponential distribution.

In the following, we will specify how the flexible transmitting scheme behaves when the channel is sensed to be busy and idle. When sensing decision of CR user is busy, CR user will back off instead of transmitting the data, therefore, there will not be collision. On the contrary, when sensing decision of CR user is idle, CR user will transmit data with exponential distribution. It is inevitable that PU will return when CR user is transmitting. Consequently, PU will suffer from the collision. Furthermore, the collision will impact the QoS of PU.

When CR user is transmitting data, there are two cases of collision to PU which is shown in Fig.1: 1) PU is busy but the sensing decision is idle due to imperfect sensing, hence when CR user is transmitting data, PU will be interfered inevitably; 2) when PU is idle and the sensing decision is idle, but the collision still maybe occur when PU returns to the ON state when CR user is transmitting.

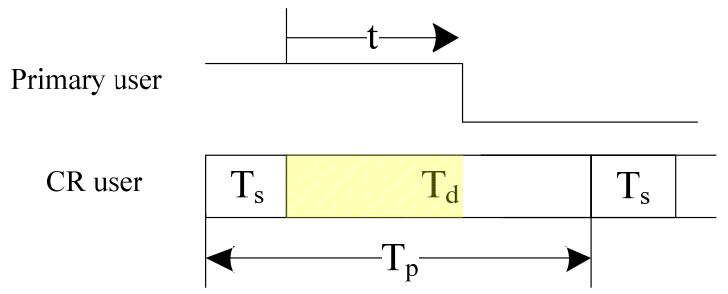

(a) Collision case 1

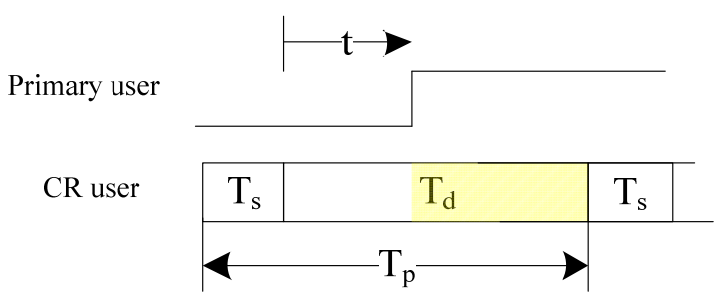

(b) Collision case 2

Figure 1. Two cases of collision to PU

In Fig. 1, $t$ denotes the time which begin with data transmission and end with the change of PU's state. The yellow part means the collision time. In special, $T_{b c}$ and $T_{i c}$ denote the collision time due to CR user's imperfect sensing and PU's return as shown in Fig.1(a) and Fig.1(b), respectively. Apparently, the longer the transmitting time, the higher the throughput of cognitive radio networks. However, longer transmitting time also leads to the higher probability of collision to PU.

In general, the objective of cognitive radio networks is to maximizing the throughput of CR users while guarantying the interference to PU above the PU can endure. However, due to PU has the priority to spectrum, it is crucial issue to ensure that the QoS of PU. Therefore, in order to guarantee the QoS of PU, we are interested in minimizing the probability of collision while maintaining the probability of detection above a certain lower bound.

Consequently, the transmitting-collision tradeoff problem can be formulated as

$$
\begin{aligned}
& \min P_{c}=\frac{T_{c}}{T_{d}} \\
& \text { s.t. } P_{d} \geq P_{d}^{*}
\end{aligned}
$$

where $P_{c}$ is the probability of collision from CR user, $T_{c}$ is the total collision time from $\mathrm{CR}$ user to $\mathrm{PU}$ under two cases mentioned above. $P_{d}$ is the probability of detection, $P_{d}{ }^{*}$ is the objective probability of detection that the $\mathrm{CR}$ user ought to obtain.

Based on two cases of collision mentioned in Fig.1, the collision time $T_{c}$ is shown in (2):

$$
T_{c}=P\left(H_{1}\right) \cdot\left(1-P_{d}\right) \cdot T_{b . c}+P\left(H_{0}\right) \cdot\left(1-P_{f}\right) \cdot T_{i . c}
$$

where $P_{f}$ is denoted by probability of false alarm, which means CR user detects the existence of PU while PU is not exist in fact. $P\left(H_{0}\right)$ and $P\left(H_{l}\right)$ mean the probability of PU's idle and busy state, respectively.

\section{PROBABILITY OF COLLISION FOR FIXED AND FLEXIBLE TRANSMITTING APPROACH}

In this section, we first review the spectrum sensing technology based on energy detection. Then we derive the probability of collision for conventional fixed frame duration scheme and proposed flexible data transmitting approach, respectively.

\section{A. Spectrum Sensing based on Energy Detection}

Spectrum sensing is a key technology for cognitive radio due to utilize the spectrum and protect the PUs. Therefore, we will study the sensing performance based on energy detection for it is widely used for spectrum sensing scheme. Let $N$ be the number of samples. The local spectrum sensing is to decide between the following two hypotheses,

$$
x(t)= \begin{cases}n(t) & \mathrm{H}_{0} \text { (primary user is absent) } \\ s(t)+n(t) & \mathrm{H}_{1} \text { (primary user is present) }\end{cases}
$$

where $x(t)$ is the observed signal at the CR user with variance 
$\sigma_{x}^{2}, s(t)$ is the PU's signal. The noise $n(t)$ is assumed to be independent and zero-mean with the Circularly Symmetric Complex Gaussian distribution $C N\left(0, N_{0}\right)$. The test statistic $Y$ for the energy detector is then given by

$$
Y=\frac{1}{N} \sum_{t=1}^{N}|x(t)|^{2}
$$

When $N$ is larger enough, the output of energy detector $Y$ follows the distribution as follows:

$$
Y- \begin{cases}x_{2 T W}^{2} & H_{0} \\ x_{2 T W}^{2}(2 \delta) & H_{1}\end{cases}
$$

where $\chi_{2 T W}^{2}$ and $\chi_{2 T W}^{2}(2 \delta)$ represent central and conditionally non-central chi-square distribution with $2 T W$ degrees of freedom, respectively. $2 \delta$ is a non-centrality parameter for the latter distribution. $\delta$ represents instantaneous signal to noise ratio (SNR) and for simplicity we assume that time-bandwidth product, $T W$, is an integer number which is denoted by $m$.

An approximate expression for the probability of detection $P_{d}$ and the probability of false alarm $P_{f}$ over Additive White Gaussion Noise (AWGN) for the energy detection can be given respectively,

$$
\begin{gathered}
P_{d}=P\left(Y>\lambda \mid H_{1}\right)=Q_{m}(\sqrt{2 \delta}, \sqrt{\lambda}) \\
P_{f}=P\left(Y>\lambda \mid H_{0}\right)=\frac{\Gamma(m, \lambda / 2)}{\Gamma(m)}
\end{gathered}
$$

where $Q_{m}(a, b)$ is the generalized Marcum Q-function given by $Q_{m}(a, x)=\frac{1}{a^{m-1}} \int_{x}^{\infty} t^{m} e^{-\frac{t^{2}+a^{2}}{2}} I_{m-1}(a t) d t$, and $\lambda$ stands for decision threshold. $\Gamma(a, x)$ is incomplete gamma function given by $\Gamma(a, x)=\int_{x}^{\infty} t^{a-1} e^{-t} d t, \Gamma(a)$ is the complete gamma function.

\section{B. Collision Analysis of Conventional Fixed Frame Duration Scheme}

Due to PU's spectrum usage state is modeled as ON/OFF model, let the duration of PU's ON and OFF states are denoted by independent and identically distributed random variables $X$ and $Y$, whose probability density functions are $f_{X}(t)$ and $f_{Y}(t)$. Usually, PU's idle and busy periods are approximated by continuous time Markov chain with the exponential distribution. Therefore, we take the assumption that the busy and idle periods follow the exponential parameters with $\alpha$ and $\beta$, whose probability density functions are

$$
f_{X}(t)= \begin{cases}\alpha \exp (-\alpha t) & \mathrm{t}>0 \\ 0 & \mathrm{t} \leq 0\end{cases}
$$

and

$$
f_{Y}(t)= \begin{cases}\beta \exp (-\beta t) & \mathrm{t}>0 \\ 0 & \mathrm{t} \leq 0\end{cases}
$$

respectively.

The average busy and idle periods are $1 / \alpha$ and $1 / \beta$. Furthermore, the stationary distributions of idle and busy periods are $P\left(H_{0}\right)=\frac{\alpha}{\alpha+\beta}$ and $P\left(H_{1}\right)=\frac{\beta}{\alpha+\beta}$, respectively.

For the fixed frame duration scheme, the collision from CR user is only related to PU's state. Based on two cases of collision mentioned above in Fig.1, $T_{b . c}$ and $T_{i . c}$ can be calculated as in (10) and (11).

$$
\begin{aligned}
& T_{b . c}=\int_{0}^{T_{d}} t f_{X}(t) d t+\int_{T_{d}}^{\infty} T_{d} f_{X}(t) d t \\
& =\int_{0}^{T_{d}} t \alpha e^{-\alpha t} d t+\int_{T_{d}}^{\infty} T_{d} \alpha e^{-\alpha t} d t=T_{d}-\frac{1}{2} \alpha T_{d}{ }^{2} \\
& T_{i . c}=\int_{0}^{T_{d}}\left(T_{d}-t\right) f_{Y}(t) d t=\int_{0}^{T_{d}}\left(T_{d}-t\right) \beta e^{-\beta t} d t \\
& =\int_{0}^{T_{d}} \beta T_{d} e^{-\beta t} d t-\int_{0}^{T_{d}} t \beta e^{-\beta t} d t=\frac{1}{2} \beta T_{d}^{2}
\end{aligned}
$$

\section{Collision Analysis of Proposed Flexible Transmitting} Scheme

In fact, CR user does not always need spectrum to transmit. Therefore, it is not reasonable to assume that CR user is always transmitting data for the fixed frame duration scheme. Consequently, in order to avoid the collision to PU, the traffic model of CR user is also need to be considered. Here, let the duration of CR user's transmitting state is denoted by random variable $Z$. We assumed that the CR user's traffic model follows an exponential distribution with a parameter $\gamma$. Hence, the probability density function is expressed as:

$$
f_{z}(t)= \begin{cases}\gamma \exp (-\gamma t) & \mathrm{t}>0 \\ 0 & \mathrm{t} \leq 0\end{cases}
$$

We assumed that the activities of PU and CR user are independent. Therefore, the joint probability density functions of $t_{l}$ and $T_{d}$ are:

$$
\begin{aligned}
& f_{i . b}\left(t, T_{d}\right)=f_{Y}(t) \cdot f_{Z}\left(T_{d}\right)=\beta \gamma \exp \left(-\beta t-\gamma T_{d}\right) \\
& f_{b . b}\left(t, T_{d}\right)=f_{X}(t) \cdot f_{Z}\left(T_{d}\right)=\alpha \gamma \exp \left(-\alpha t-\gamma T_{d}\right)
\end{aligned}
$$

where $f_{i . b}\left(t, T_{d}\right)$ denotes the joint probability density function 
when PU is idle and CR user is busy, and $f_{b . b}\left(t, T_{d}\right)$ denotes the joint probability density function when PU is busy and CR user is busy.

In the flexible transmitting scheme, the collision to PU is not only related to PU's state, but also have the relationship with the state of CR user. Let $T_{b . c}{ }^{*}$ and $T_{i . c}{ }^{*}$ denote the collision time with two cases when the CR user is transmitting. Then $T_{b . c}{ }^{*}$ and $T_{i . c}{ }^{*}$ can be calculated as (15) and (16), respectively.

$$
\begin{aligned}
& T_{b . c}{ }^{*}=\int_{0}^{T_{d}} t f_{b . b}\left(t, T_{d}\right) d t+\int_{T_{d}}^{\infty} T_{d} f_{b . b}\left(t, T_{d}\right) d t \\
& =\int_{0}^{T_{d}} t \alpha \gamma e^{-\alpha t-\gamma T_{d}} d t+\int_{T_{d}}^{\infty} T_{d} \alpha \gamma e^{-\alpha t-\gamma T_{d}} d t \\
& =r e^{-r T_{d}} \cdot \int_{0}^{T_{d}} \alpha t e^{-\alpha t} d t+T_{d} r e^{-r T_{d}} \int_{T_{d}}^{\infty} \alpha e^{-\alpha t} d t \\
& =\gamma T_{d}-\frac{1}{2}\left(2 \gamma^{2}+\alpha \gamma\right) T_{d}{ }^{2} \\
& T_{i . c}{ }^{*}=\int_{0}^{T_{d}}\left(T_{d}-t\right) f_{i . b}\left(t, T_{d}\right) d t \\
& =\int_{0}^{T_{d}}\left(T_{d}-t\right) \beta \gamma e^{-\beta t-\gamma T_{d}} d t=\frac{1}{2} \beta \gamma T_{d}^{2}+\frac{1}{2} \gamma^{3} \beta^{3}
\end{aligned}
$$

Therefore, by substituting (15) and (16) into (2) and (1), the probability of collision $P_{c}{ }^{*}$ from CR users for flexible transmitting scheme can be expressed as in (17).

$$
\begin{aligned}
& P_{c}^{*}=\frac{T_{c}{ }^{*}}{T_{d}} \\
& =\frac{P\left(H_{1}\right) \cdot\left(1-P_{d}\right) \cdot\left[\gamma T_{d}-\frac{1}{2}\left(2 \gamma^{2}+\alpha \gamma\right) T_{d}^{2}\right]}{T_{d}} \\
& +\frac{P\left(H_{0}\right) \cdot\left(1-P_{f}\right) \cdot\left(\frac{1}{2} \beta \gamma T_{d}^{2}+\frac{1}{2} \gamma^{3} \beta^{3}\right)}{T_{d}}
\end{aligned}
$$

In order to compare the performance with the fixed frame duration approach, by substituting (10) and (11) into (2) and (1), the probability of collision $P_{c}$ for the conventional scheme can be derived as in (18).

$P_{c}=\frac{T_{c}}{T_{d}}=\frac{P\left(H_{1}\right) \cdot\left(1-P_{d}\right) \cdot\left(T_{d}-\frac{1}{2} \alpha T_{d}^{2}\right)+P\left(H_{0}\right) \cdot\left(1-P_{f}\right) \cdot \frac{1}{2} \beta T_{d}^{2}}{T_{d}}$

\section{NUMERICAL SIMULATION}

In this section, we provide the numerical results for the transmitting-collision tradeoff problem under the probability of detection constrains by using MATLAB simulation tool. In the following, we set the target probability of detection $\mathrm{P}_{\mathrm{d}}{ }^{*}=0.9$. Assumption that the duration of busy and idle state of PU follow an exponential distribution with parameter $\alpha=0.4$ and $\beta=0.2$.

Fig. 2 presents the performance comparison between fixed frame duration approach and flexible data transmitting scheme when the probability of detection $P_{d}$ is 0.95 and probability of false alarm $P_{f}$ is 0.1 . CR user follows an exponential distribution with a parameter $\gamma=0.2$. The results show that with the increasing of data transmitting time $T_{d}$, the probability of collision is increasing gradually. Moreover, the probability of collision for proposed scheme is remarkable lower than the conventional approach.

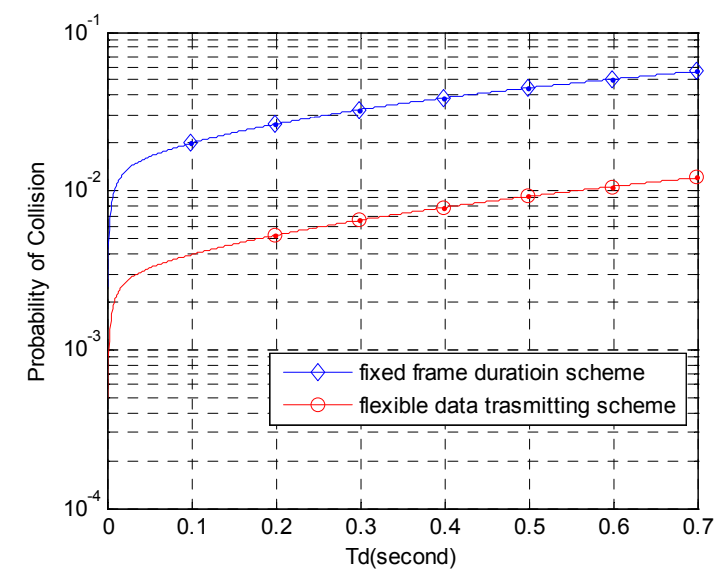

Figure 2. The comparion of collision between proposed and conventional scheme

After analyzing the superiority of presented scheme, we investigate the impact from traffic parameter of CR user and detection performance metrics, respectively. Fig. 3 shows the collision impact from traffic parameter of CR user $\gamma$ to the PU. The larger the traffic parameter $\gamma$, the higher the probability of collision. The reason for that the larger traffic parameter means CR user needs the more chances to occupy the spectrum, therefore, it leads to the higher probability of collision.

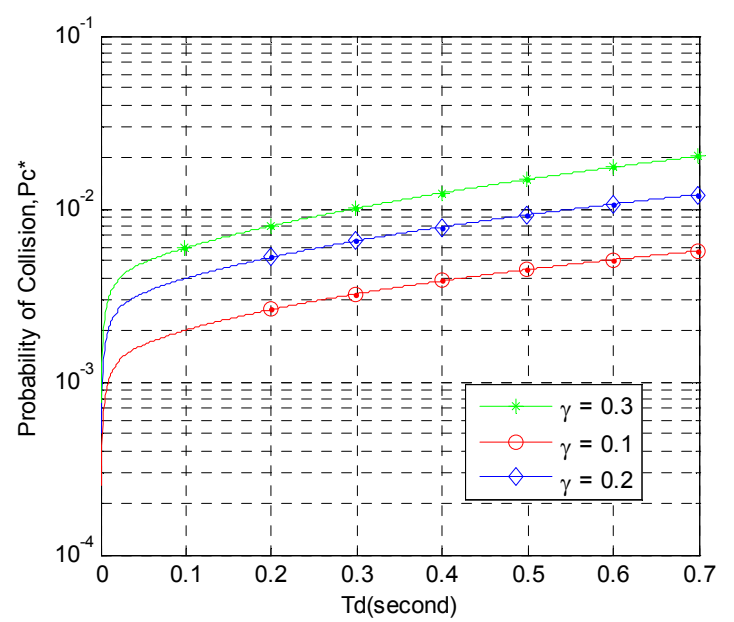

Figure 3. The impact from traffic parameter on probability of collision for the proposed scheme

Fig.4 demonstrates that the probability of collision 
decreased with the increasing of the probability of detection $P_{d}$. The reason for that the larger probability of detection means CR user could sense the occupied spectrum correctly. Therefore, the collision to the PU can be avoided due to the good sensing performance.

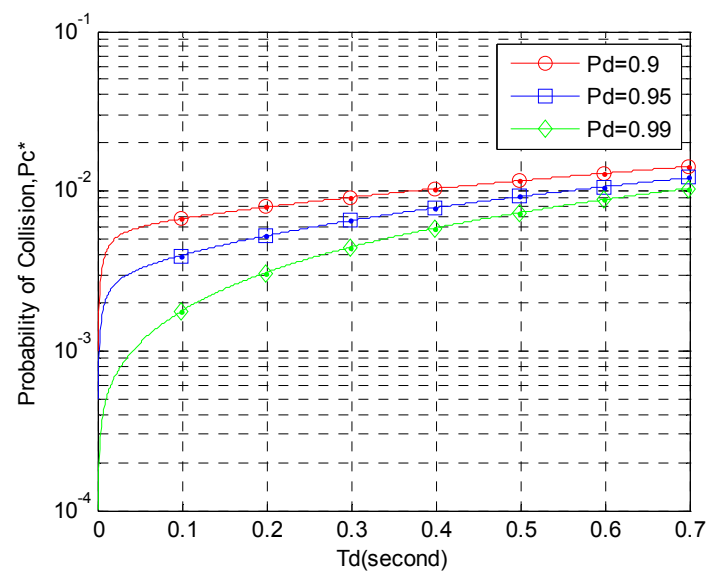

Figure 4. The impact from probability of detection on probability of collision for the proposed scheme

\section{CONCLUSIONS}

In this paper, we have investigated the flexible data transmitting scheme based on jointly considering the traffic model of PU and CR user. The proposed scheme aims to minimize the collision to PU under the probability of detection constrains. The simulation results indicate that the probability of collision of the proposed scheme is less than conventional approach obviously. Besides, we investigate the impact from traffic parameter and detection performance for the presented approach. The results show that the larger traffic parameter of CR user leads to higher collision to PU. And higher probability of detection brings about lower collision to PU.

\section{ACKNOWLEDGEMENT}

This work is supported by the Fundamental Research Funds for the Central Universities.

\section{REFERENCES}

[1] Federal Communications Commission, "Spectrum Policy Task Force, Rep. ET Docket no. 02-135,” Nov. 2002.

[2] S. Haykin, "Cognitive radio: Brain-empowered wireless communications," IEEE J. Sel. Areas Commun., vol. 23, no. 2, Feb. 2005.pp. 201-220

[3] Y-C Liang, Y. Zeng, E. C. Y. Peh, and A. T. Hoang, "Sensing-throughput tradeoff for cognitive radio networks," IEEE Trans. Wireless Commun, vol. 7, Apr. 2008, pp. 1326-1337.

[4] Karama Hamdi and Khaled Ben Letaief. "Power, Sensing Time, and throughput Tradeoffs in Cognitive Radio Systems: A Cross-Layer Approach," Proc. of IEEE Wireless Comm. and Networking Conf., 2009, pp: $1-5$

[5] Edward C. Y. Peh, Ying-Chang Liang and Yong Liang Guan. "Optimization of Cooperative Sensing in Cognitive Radio Networks: A Sensing-Throughput Tradeoff View," Proc. of IEEE International Conference on Communications, 2009, pp:1-5

[6] Xin Li, Qianchuan Zhao, Xiaohong Guan and Lang Tong. "Sensing and Communication Tradeoff for Cognitive Access of Continues-Time Markov Channels," proc. of IEEE Wireless Comm. and Networking Conf, 2010, pp:1-6
[7] Edward Chu Yeow Peh, Ying-Chang Liang, Yong Liang Guan and Yonghong Zeng, "Optimization of Cooperative Sensing in Cognitive Radio Networks: A Sensing-Throughput Tradeoff View," IEEE TRANSACTIONS ON VEHICULAR TECHNOLOGY, VOL. 58, NO. 9, NOVEMBER 2009, pp:5294-5299

[8] Yiyang Pei, Ying-Chang Liang, Kah Chan Teh and Kwok Hung Li. "Sensing-Throughput Tradeoff for Cognitive Radio Networks: A Multiple-Channel Scenario," proc. of IEEE 20th International Symposium on Personal, Indoor and Mobile Radio Communications, 2009, pp:1257-1261

[9] Young-June Choi, Yan Xin, and Sampath Rangarajan. "Overhead-Throughput Tradeoff in Cooperative Cognitive Radio Networks," proc. of IEEE Wireless Comm. and Networking Conf., 2009, pp:1-6

[10] Alexander W. Min and Kang G. Shin. "On Sensing-Access Tradeoff in Cognitive Radio Networks," proc. of IEEE Symposium on New Frontiers in Dynamic Spectrum, 2010, pp:1-12

[11] Yu-long Zou, Yu-Dong Yao and Bao-yu Zheng, "Spectrum sensing and data transmission tradeoff in cognitive radio networks," Proc. of Wireless and Optical Communications Conference, 2010, pp: 1-5.

[12] Stergios Stotas and Arumugam Nallanathan, "Overcoming the Sensing-Throughput Tradeoff in Cognitive Radio Networks," Proc. of IEEE International Conference on Communications, 2010, pp:1-5

[13] Li Hong-jiang and Zhu Qi, "Tradeoff between Utilization and Collision in Cognitive Radio," Proc. of Wireless Communications \& Signal Processing, 2009, pp:1-5 